\title{
Microwave Assisted $N$-Alkenyl Condensation between Pyrrolidine-2-thione and Various Aldehydes
}

\author{
Ki-Won Kim,${ }^{\dagger}$ Ho-Joon Lee, ${ }^{\dagger}$ Chung-Gi Kim, ${ }^{\dagger}$ Mi-ja Park,,${ }^{\dagger}$ and Tae-Woo Kwon ${ }^{\dagger+{ }^{\circ}}$ \\ ${ }^{\dagger}$ Department of Chemistry and ${ }^{-}$Department of Chemical Education, Kungsung Lniversitw, Busan 608-736, Korea \\ E-mail: nikwonaks.ackr \\ Received Februarv 15, 2007
}

\begin{abstract}
A series of $N$-alkenyl py rolidine-2-thiones were sy nthesized by the reaction between pyrrolidine-2-thione and various aldehỵdes such as n-propanal, isopropanal, $n$-butanal, $n$-hexanal, $n$-octanal and phenylacetaldehyde in $32-86 \%$ yields using microwave irradiation technique. Only one stnctural $E$ isomers were predominantly formed within 15 minutes in cllorobenezene/p-toluenesulfonic acid monohydrate
\end{abstract}

Key Words : Microwave irradiation, Pyrrolidine-2-thione. $N$-Alkenyl pyrrolidine-2-thiones. $N$-Alkenyl condensation

\section{Introduction}

$N$-Vinyl analogues are widely used as very important intermediates in polymerizations. " alkaloid syntheses." protecting procedure ${ }^{3}$. Diels-Alder reactions ${ }^{4}$. regioselective Heck couplings and pyrimidine syntheses. Whereas numerous reaction methods for the preparation of $N$-vinyl analogues have been previously reported using dehydration. ${ }^{7}$ dehydroalkoxylation. ${ }^{8}$ copper $^{9}$ and palladium ${ }^{10}$ catalyzed vinylation. coupling with 2-halo vinyliodides. ${ }^{1]}$ and decomposition of acyloxy ethyl derivatives. ${ }^{2}$ introducing vinyl moieties at the nitrogen atom in lactams have not been generally studied.

Recently microwave irradiation techniques for a variety of organic reactions are of great interest. Compared to the wellknown conventional heating methods. high efficiency heating using microwave irradiation has been shown to dramatically accelerate reaction rates and reduce the reaction time with high yields. ${ }^{13}$ We have demonstrated that a variety of reactions such as Knoevenagel condensations. ${ }^{1+}$ Michael addition. ${ }^{15}$ nitration or $U$-alkylation in the aromatic rings. ${ }^{16}$ and Friedlander type quinoline synthesis ${ }^{17}$ can be facilitated by the microwave irradiation technique in the presence or absence of solvents. Although many microwave enhanced organic reactions have been explored. direct $N$-alkenylation reactions and their applications for the protection of $\mathrm{NH}$ atom in the lactam rings were poorly studied. More recently. in the course of investigation aimed at the synthesis of $\gamma$ vinyl amino butyric acid ${ }^{18}$ our prior extensive study has shown that lactam N-H 1 can be successfully condensed with several different types of aldehydes to give $N$-alkenyl analogues 2 under a conventional heating method using the Dean-Stark apparatus ${ }^{19}$ or under microwave irradiation ${ }^{15}$ conditions within a short period of time. The pyrrolidine-2thione compounds. 3 are also of synthetic value. For example. 3 can be easily transformed to the $S$-methyl $(\mathrm{R}=$ $\left.\mathrm{CH}_{3}\right)$ or $S$-benzyl $\left(\mathrm{R}=\mathrm{CH}_{2} \mathrm{Ph}\right)$ compounds 4 . by refluxing with methyl iodide in tetrahydrofuran and are also readily converted to the parent pyrrolidine-2-one. 1 in the presence of aqueous hydroiodide. Even though some alkaloids and total synthesis using $N$-alkyl thiolactams have been reported. ${ }^{20}$ syntheses and applications of $N$-alkenyl pyrrolidine-2thione 5 using microwave irradiation technique have been rarely investigated so far. In here, we wish to represent the first successful application of microwave irradiation for the reaction between pyrrolidine-2-thione. 3 and a variety of aldehy'des.

\section{Results and Discussion}

Pỵrrolidine-2-thione. 3 was șinthesized by refluxing of pyrrolidine-2-one. 1 and Lawesson's reagent in toluene as previously described in the literature. ${ }^{21}$ We initially exanined eco-friendly $N$-alkenylation of 3 in the absence of solvent using a conventional microwave oven. However, these reactions were not successful and the yield from GC-MS analy sis showed as low as $0-5 \%$ in $\mathrm{K}_{10}$ or bentonite in $\mathrm{Al}_{2} \mathrm{O}_{3}$ (Table 1, entries 1-5). Using solvent for the condensation. the yields were significantly improved in our experimental conditions. It was found that cllorobenzene was the best

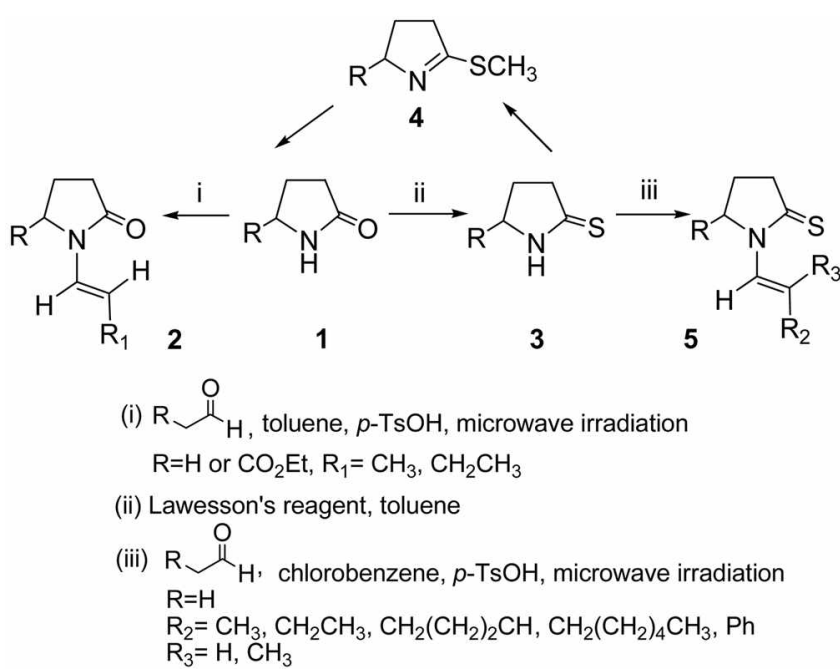

Scheme 1 
Table 1. Microwave assisted N-alkenvl condensation of lactam-2thione, 3 with aldehydes using different solid supports and solvents (inadiation time: 15 min)

\begin{tabular}{ccccc}
\hline Entry & aldehyde & $\begin{array}{c}\text { catalyst } \\
\text { /solid support }\end{array}$ & solvent & yield $(\%)^{b}$ \\
\hline 1 & hexanal & none $/ \mathrm{Al}_{2} \mathrm{O}_{3}$ & none & 0 \\
2 & hexanal & $\mathrm{K}_{10} / \mathrm{Al}_{2} \mathrm{O}_{3}$ & none & 0 \\
3 & hexanal & none $/$ bentonite & none & 5 \\
4 & octanal & $\mathrm{K}_{10} / \mathrm{Al}_{2} \mathrm{O}_{3}$ & none & 3 \\
5 & octanal & $p$-TsOH/none & none & 0 \\
6 & octanal & $p$-TsOH/none & $m$-xylene & $47^{\circ}$ \\
7 & octanal & $p$-TsOH/none & chlorobenzene & $59^{\circ}$ \\
\hline
\end{tabular}

"Mole ratio of 3 : aldeliydes $=1: 1.5$. "Yields based on quantitative GCMS analysis. 'Isolated vields based on starting pyrrolidin-2-thiones after purification

choice as an energy-transfer medium since its boiling point $\left(131.133^{\circ} \mathrm{C}\right)$ is about $30^{\circ} \mathrm{C}$ higher than water, which is to be eliminated in the process. When the reaction was performed in $m$-xylene and chlorobenzene, we obtained 47 and $59 \%$ yields (Table 1 , entries 6 and 7 ) respectively which were remarkably higher than using other solvents such as benzene or toluene. To avoid a solvent pressure in the reaction vessel, a large vial with a loose cap or an erlenmeyer flask with a fumnel as a loose top was used. The microwave irradiation was carried with 3 minutes intervals and addition of chlorobenzene $(1-3 \mathrm{~mL})$ was necessary due to slight solvent evaporation.

This reaction condition is directly applicable to the synthesis for a variety of $\mathrm{N}$-alkenyl thiolactams. As shown in Table 2, a series of $N$-alkenyl pyrrolidine-2-thiones were successfully synthesized by the microwave assisted condensation of pyrrolidin-2-thione with $n$-propanal. isopropanal, $n$-butanal. $n$-hexanal. n-octanal and phenylacetaldehyde. The results of our studies suggest a strong dependence of boiling point of aldehydes. As the boiling point of aldelydes increased. greater yields were obtained. This is probably due to the lower evaporating loss of higher boiling aldehydes in

Table 2. N-alkenyl condensation of lactam-2-thione, 3 with various aldehydes under conventional heating versus microwave assisted inradiation (MWI)

\begin{tabular}{|c|c|c|c|c|c|}
\hline \multirow{2}{*}{ Entry } & \multirow{2}{*}{ Aldehyde ${ }^{\sigma}$} & \multirow{2}{*}{ Product ${ }^{b}$} & \multirow{2}{*}{$\begin{array}{c}\mathrm{Rf} \\
\left(100 \% \% \mathrm{CH}_{2} \mathrm{Cl}_{2}\right)\end{array}$} & \multicolumn{2}{|c|}{ Yield $(\%)^{\circ}$} \\
\hline & & & & $\Delta^{d}$ & MWI $^{e}$ \\
\hline 1 & $\mathrm{CH}_{3} \mathrm{CH}_{2} \mathrm{CHO}$ & & 0.60 & 6 & 32 \\
\hline 2 & $\left(\mathrm{CH}_{3}\right)_{2} \mathrm{CHCHO}$ & & $0.52^{f}$ & - & 36 \\
\hline 3 & $\mathrm{CH}_{3} \mathrm{CH}_{2} \mathrm{CH}_{3} \mathrm{CHO}$ & & 0.57 & 5 & 51 \\
\hline 4 & $\mathrm{CH}_{3}\left(\mathrm{CH}_{2}\right)_{2} \mathrm{CH}_{2} \mathrm{CHO}$ & & 0.77 & 5 & 41 \\
\hline 5 & $\mathrm{CH}_{3}\left(\mathrm{CH}_{2}\right)_{3} \mathrm{CH}_{2} \mathrm{CHO}$ & & $0.37^{g}$ & - & 59 \\
\hline 6 & $\mathrm{PhCH}_{2} \mathrm{CHO}$ & & $0.68^{\prime \prime}$ & - & 86 \\
\hline
\end{tabular}

"Mole ratio of pyrrolidin-2-thiones:aldehyde $=1: 1.5$. solvent:chlorobenzene). "Catalyst: $p$-TsOH. reaction time: 15 min. 'Isclated yields based on

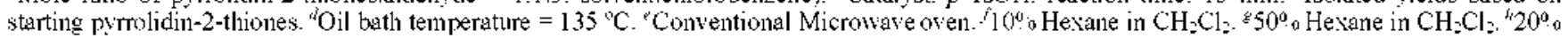
Hexane in $\mathrm{CH}_{2} \mathrm{Cl}$.

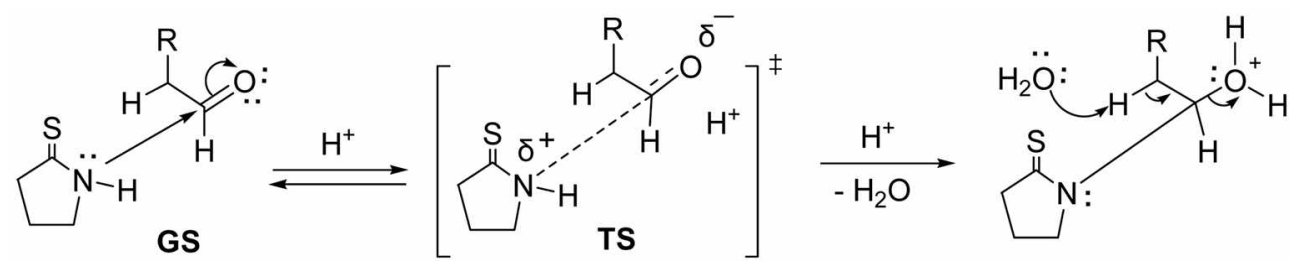

Figure 1. Proposed mechantism for the ground state (GS) versus dipole-dipole electrostatic transition state (TS) between pynolidin-2-thione and aldehyde. 
<smiles>[R]C1=C(C)N2COC[C@@]([2H])([C@@H]([2H])C2=S)C1(C)C</smiles>

E12. $\mathrm{R}=\mathrm{CH}_{2}\left(\mathrm{CH}_{2}\right)_{4} \mathrm{CH}_{3}$

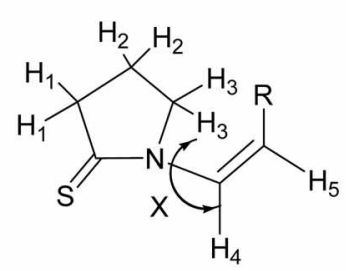

Z14. $\mathrm{R}=\mathrm{CH}_{2}\left(\mathrm{CH}_{2}\right)_{4} \mathrm{CH}_{3}$

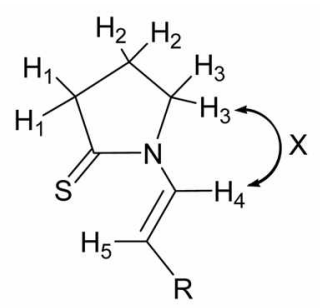

E13. $\mathrm{R}=\mathrm{CH}_{2}\left(\mathrm{CH}_{2}\right)_{4} \mathrm{CH}_{3}$

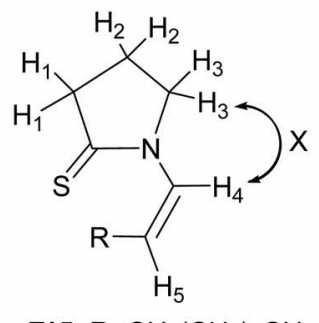

Z15. $\mathrm{R}=\mathrm{CH}_{2}\left(\mathrm{CH}_{2}\right)_{4} \mathrm{CH}_{3}$

Figure 2. $E(E 12$ and $E 13) / Z(Z 14$ and $Z 15)$ alkene isomer structures and observed NOE interactions between $\mathrm{H}-3$ and $\mathrm{H}-5$ in E12.

the reaction media. In the case of phenylacetaldehyde $(\mathrm{bp}=$ $195^{\circ} \mathrm{C}$ ). we obtained the highest yield as a $91 \%$ (entry 6). All thiolactam alkenyl products $(6,7.8 .9$ and 10$)$ were isolated as an oil form except the phenylacetaldehyde derivative $\left(11, \mathrm{mp}=118-120^{\circ} \mathrm{C}\right)$.

The experimental results using microwave irradiation (MWI) and conventional heating were compared to see nonthermal MW effects. The same reaction conditions like vessel temiperature. reaction time and mole ratio used under MW were performed in the conventional heating experiments using a preheated oil bath (Table 2 , entries 1,3 . and 4). In all cases, conventional heating gave considerably low yields which clearly proves the efficacy of the specific and non-thermal MW effects. It has been emphasized that specific MW effects were generally observed for polar mechanism from the ground state (GS) towards the transition state (TS) when the polarity increased. ${ }^{13 .{ }^{3} 3}$ As shown in the proposed mechanism (Figure 1), the formation of $\mathrm{N}$ alkenyl product (5) was accelerated by microwave irradiation due to the electric field induced dipole-dipole intermediate in the rate-determining step. The intermolecular nucleophilic addition of lactam nitrogen to the aldehyde carbonyl carbon in the GS involves the formation of an electrostatic polarized TS which is stabilized under microwave conditions. Further elimination of 1 equivalent of water gave $N$-alkenylated products (5) in an mild acidic medium

$E Z Z$ alkene isomers can be often detemined on the basis of large coupling constants $(J)$ as ascertained by ' ${ }^{2} \mathrm{H}$ NMR. Large coupling constants of $E$ alkenyl hydrogens of 6 at $\delta$ $7.54(J=13.05 \mathrm{~Hz}), 8$ at $\delta 7.49(J=14.5 \mathrm{~Hz}), 9$ at $\delta 7.48(J$ $=14.46 \mathrm{~Hz}), 10$ at $\delta 7.48(J=14.58 \mathrm{~Hz})$ and 11 at $\delta 8.27(J=$ $15.03 \mathrm{~Hz})$ ppm were observed respectively in the ${ }^{1} \mathrm{H}$ NMR. We have also performed two-dimensional NOESY experi-

ments to evaluate the neighborhood proton influence. Figure 2 shows four possible $E$ or $Z$ isomer structures $(E 12, E 13$, $Z 1+$ and $Z 15$ ) of octenyl thiolactam (10). Interacting NOE cross peaks between $\mathrm{H}-3$ on the thio-lactam ring and the closest proton $\mathrm{H}-5$ of the alkenyl moiety were observed as shown in $E 12$. No cross peaks corresponding to the proton interactions between $\mathrm{H}-3$ and $\mathrm{H}-4$ were observed (E13. Z14 and $Z 15$ ). We thus determined that $E$ isomers were predominantly produced in all cases (Table 3 . entries $1,3,4,5$ and 6). Only trace amount of $Z$ isomers $(1-2 \%>)$ were detected in the crude ${ }^{1} \mathrm{H}$ numr spectra.

\section{Summary}

This presentation describes a fast and facile microwave enhanced $N$-alkenylation synthesis between sulfur-containing lactam and various aldelyydes such as $n$-propanal. isopropanal. $n$-butanal, $n$-hexanal, $n$-octanal and phenylacetaldehyde in 32 to $86 \%$ yields. In order to explain the conformational properties of $E Z$ isomers, we used the coupling constant $(J)$ of ${ }^{1} \mathrm{H}$ and 2-D NOESY NMR techniques. In all cases, $F$ isomers were predominantly produced. We expect these new $N$-alkenyl pyrrolidine-2-thiones to have interesting applications for the protection of functionalized thiolactams. Consequently, we are currently expanding our results using the advantages of microwave irradiation.

\section{Experimental Section}

Pyrrolidin-2-one, Lawesson's reagent, $p$-toluenesulfonic acid monohydrate $(98.5 \%)$, bentonite, $\mathrm{K}_{10} \mathrm{Al}_{2} \mathrm{O}_{3}, n$-propanal, isopropanal. $n$-butanal, $n$-hexanal. $n$-octanal and phenylacetaldelyyde were purchased from Sigma-Aldrich (Korea) and used as received. Toluene. chlorobenzene and $m$-xylene were either used as supplied or purified by standard tecluniques. TLC was performed on precoated glass plate-silica gel $250-\mu \mathrm{m}$ (Baker $\mathrm{Si} 250 \mathrm{~F}$ ) with detection by UV light. Flash column chromatography was perfonmed on silica gel $\left(230-400\right.$ mesh). The ${ }^{1} \mathrm{H}$ and ${ }^{13} \mathrm{C}$ NMR spectra were recorded at $300 \mathrm{MHz}$ and $75 \mathrm{MHz}$ on a FT-NMR Bnuker 300. Chemical shifts are given in ppm and referenced to intemal tetramethylsilane (TMS, $\delta=0 \mathrm{ppm}$ ) standard. GC/MS spectra were measured on a Shimazu QP 5000 spectrometer.

Pyrrolidin-2-thione, 3. To a $500 \mathrm{~mL}$ round-bottomed flask equipped with a mechanical stirrer, reflux condenser and a nitrogen line was charged $2(5.2 \mathrm{~g} .61 .2 \mathrm{mmol}$. 1 equiv.). powdered Lawesson's reagent ( $24.7 \mathrm{~g} .61 .2 \mathrm{mmol}$. 1 equiv.) and toluene $(200 \mathrm{~mL})$ were added. The mixture was refluxed under $\mathrm{N}_{2}$ atmosphere. After 4 lur. the starting material was detemined to be disappeared by TLC analysis $\left(2 \% \mathrm{MeOH} / 98 \% \mathrm{CH}_{2} \mathrm{Cl}_{2}\right)$. The removal of the solvent using rotary evaporator under reduced pressure and flash colunn chromatography using $2 \% \mathrm{MeOH} / 98 \% \mathrm{CH}_{2} \mathrm{Cl}_{2}$ as eluent afforded the product, 3 (4.6 g. 45.9 nmole, $75 \%$ ) as a solid. mp 112-114 ${ }^{\circ} \mathrm{C}$ (lit ${ }^{\prime} 114-115^{\circ} \mathrm{C}$ ), ' $\mathrm{H}$ NMR ( $\mathrm{CDCl}_{3} .300$ MHz) $\delta 8.7 \mathrm{l}$ (br s. $1 \mathrm{H}) .3 .67$ (t. $J=7.2 \mathrm{~Hz}, 2 \mathrm{H}), 2.84(\mathrm{t}, J=$ 
$7.9 \mathrm{~Hz} .2 \mathrm{H}) .2 .13$ (m. $2 \mathrm{H}$ ). ${ }^{13} \mathrm{C}$ NMR $\left(\mathrm{CDCl}_{3}, 75 \mathrm{MHz}\right) \delta$ $205.52,49.73,43.38$ and $22.87 \mathrm{ppm}$. IR (neat) 3420,3200 . 2952. 1540,1475 and $1349 \mathrm{~cm}^{-1}$

The typical experimental procedure for the synthesis of (E)-1-(but-1-enyl)pyrrolidine-2-thione, 8 (Table 3. entry 3 ) is as follows: The reaction vessel containing pyrrolidine-2-thione, 3 (100 mg. 0.99 munol, I equiv), n-butanal (107 $\mathrm{mg} .1 .49 \mathrm{mmol} 1.5$ equiv) and $98.5 \%$ p-toluenesulfonic acid monohydrate $(5 \mathrm{mg})$ in chlorobenzene $(10 \mathrm{~mL})$ were placed in a $2450 \mathrm{MHz}$ Samsung microwave oven (model \# RE-555 TCW). The mixture was irradiated 5 times for 15 $\mathrm{min}$ with $3 \mathrm{~min}$ intervals. Additional chlorobenzene $(3 \mathrm{~mL})$ was repeatedly provided in the reaction vessel after 3 minutes of microwave irradiation. After completion of the reaction. the mixture was allowed to cool at the room temperature, and the crude product was extracted into $\mathrm{CH}_{2} \mathrm{Cl}_{2}$. The removal of the solvent using rotary evaporator under reduced pressure and flash colunun chromatography using $\mathrm{CH}_{2} \mathrm{Cl} / \mathrm{MeOH}(98 / 2)$ as eluent afforded the product. 8 (78.2 $\mathrm{mg}, 0.50 \mathrm{mmol}, 5 \mathrm{l} \%$ ). ${ }^{\mathrm{H}} \mathrm{H} \mathrm{NMR}\left(300 \mathrm{MHz} . \mathrm{CDCl}_{3}\right): \delta$ 7.49 (lH. d. $J=14.5$ Hz). 5.38 (lH. m). 3.79 (2H. t. $J=7.56$ $\mathrm{Hz}), 3.06(2 \mathrm{H}$. t. $J=7.92 \mathrm{~Hz}), 2.12(4 \mathrm{H} . \mathrm{m})$ and $1.04(3 \mathrm{H}, \mathrm{t}$. $J=7.41 \mathrm{~Hz}){ }^{13} \mathrm{C}$ NMR $\left(75 \mathrm{MHz}, \mathrm{CDCl}_{3}\right): \delta 199.60,126.53$. $121.36,52.85,45.70 .23 .83 .19 .84$ and $14.51 \mathrm{ppm}$. Infrared (neat): 2962.9-2861.2, 1655.1, 1483.1. 1433.8. 1420.1, 1336.9. $1294.9,1279.5,1136.4$ and $952.0 \mathrm{~cm}^{-1}$. GC/MS $155\left(\mathrm{M}^{+}\right.$. $25) .140(3) .126(100), 112(2), 98(20), 85(16)$ and $68(7)$. MALDI-TOF-MS $\mathrm{m} / \mathrm{z}$ calcd for $\mathrm{C}_{8} \mathrm{H}_{13} \mathrm{NS} /$ Exact Mass: $155.0769 /$ found 155.1592 .

(E)-1-(Prop-1-enyl)pyrrolidine-2-thione, 6 (Table 3, entry 1) ${ }^{\mathrm{H}} \mathrm{H}$ NMR $\left(300 \mathrm{MHz}, \mathrm{CDCl}_{3}\right): \delta 7.54(\mathrm{lH}, \mathrm{d} . J=$ $13.05 \mathrm{~Hz}) .5 .39(\mathrm{lH} . \mathrm{m}) .3 .82(2 \mathrm{H} . \mathrm{t} . J=7.11 \mathrm{~Hz}) .3 .09(2 \mathrm{H}$. t. $J=7.74 \mathrm{~Hz}) .2 .12(2 \mathrm{H}, \mathrm{m})$ and $1.80(3 \mathrm{H} . \mathrm{d} . J=6.84 \mathrm{~Hz})$. ppm. ${ }^{13} \mathrm{C}$ NMR (75 MHz. $\left.\mathrm{CDCl}_{3}\right): \delta 199.51,126.9,119.87$. 52.64. 45.32. 2319.61 and $18.02 \mathrm{ppm}$. GC/MS $1+1{ }^{\prime}\left(\mathrm{M}^{+}\right.$. $85) .126(100) .112(12) .98(58), 85(25) .71(32) .56(50)$ and $45(53)$. FAB MS, $\mathrm{m} / \mathrm{s} \mathrm{I} 4 \mathrm{l} .3\left(\mathrm{M}^{-}\right)$.

(E)-1-(2-Methylprop-1-enyl)pyrrolidine-2-thione, 7 (Table 3, entry 2) ${ }^{1} \mathrm{H}$ NMR (300 MHz. $\left.\mathrm{CDCl}_{3}\right): \delta 6.27(\mathrm{lH}$. s). $3.89(2 \mathrm{H}$, t. $J=7.29 \mathrm{~Hz}) .3 .04(2 \mathrm{H} . \mathrm{t} . J=3.92) .2 .58(1 \mathrm{H}$. m) $1.83(3 \mathrm{H}, \mathrm{s})$ and $1.71(3 \mathrm{H}, \mathrm{s}) \mathrm{ppm} .{ }^{13} \mathrm{C} \mathrm{NMR}(75 \mathrm{MHz}$. $\left.\mathrm{CDCl}_{3}\right): \delta 199.49,127.15$. 119.61, 52.72. 45.41, 23.01. 19.75 and $18.93 \mathrm{ppm}$. Infrared (neat): $2971.5-2913.3,1488.3$. 1457.9. 1419.3. 1327.5. 1289.8 and $1130.0 \mathrm{~cm}^{-1}$. FAB MS. $\mathrm{m} / \mathrm{s} 155.1\left(\mathrm{M}^{+}\right)$.

(E)-1-(Hex-1-enyl)pyrrolidine-2-thione, 9 (Table 3, entry 4) ${ }^{1} \mathrm{H}$ NMR $\left(300 \mathrm{MHz}, \mathrm{CDCl}_{3}\right): 7.48(\mathrm{lH} . \mathrm{d}, J=14.46 \mathrm{~Hz})$. $5.33(\mathrm{lH} . \mathrm{m}) .3 .79(2 \mathrm{H}, \mathrm{t}, J=7.44 \mathrm{~Hz}) .3 .05(2 \mathrm{H}, \mathrm{t}, J=7.89$ $\mathrm{Hz}) .2 .10(4 \mathrm{H}, \mathrm{m}) .1 .34(4 \mathrm{H}, \mathrm{m})$ and $0.86(3 \mathrm{H}, \mathrm{t} . J=6.98$ Hz) ppm. ${ }^{13} \mathrm{C}$ NMR $\left(75 \mathrm{MHz} . \mathrm{CDCl}_{3}\right): \delta 199.44,127.01$. $119.85,52.85,45.65,32.33,30.21,22.72 .19 .82$ and 14.28 ppm. Infrared (neat): $2966.5-2856.7,1650.3,1483.2,1458.5$. $1432.4 \quad 1339.5,1275.6 .1136 .9$ and $950.9 \mathrm{~cm}^{-1}$. FAB MS. $\mathrm{m} / \mathrm{s} 183.2\left(\mathrm{M}^{+}\right)$.

(E)-1-(Oct-1-enyl)pyrrolidine-2-thione, 10 (Table 3, entry 5) ${ }^{1} \mathrm{H}$ NMR (300 MHz, $\left.\mathrm{CDCl}_{3}\right): \delta 7.48(1 \mathrm{H}, \mathrm{d}, J=14.58$ $\mathrm{Hz}), 5.37(1 \mathrm{H}, \mathrm{m}), 3.78(2 \mathrm{H} . \mathrm{t} . J=7.47 \mathrm{~Hz}) .3 .05(3 \mathrm{H} . \mathrm{t} . J=$
$7.74 \mathrm{~Hz}) .2 .08(4 \mathrm{H}, \mathrm{m}) .1 .28(8 \mathrm{H}, \mathrm{m})$ and $0.85(3 \mathrm{H}, \mathrm{t}) \mathrm{ppm}$. ${ }^{13} \mathrm{C}$ NMR $\left(75 \mathrm{MHz} . \mathrm{CDCl}_{3}\right): \delta$ 199.08. 126.65. 119.47 . $52.4115 .45 .24,31.64 .30 .15,29.71 .28 .80,22.57 .19 .42$, and $14.06 \mathrm{ppm}$. Infrared (neat): $2966.5-2870.3$. 1654.1. 1483.1. $1483.3,1439.5,1419.6,1339.7 .1276 .9,1137.2$ and 952.0 $\mathrm{cm}^{-1}$. FAB MS. $\mathrm{m} / \mathrm{s} 211.1\left(\mathrm{M}^{+}\right)$

(E)-1-Styrylpyrrolidine-2-thione, 11 (Table 3, entry 6) mp 118-120 ${ }^{\circ} \mathrm{C} .{ }^{1} \mathrm{H} \mathrm{NMR}\left(300 \mathrm{MHz}, \mathrm{CDCl}_{3}\right): \delta 8.27(1 \mathrm{H}, \mathrm{d}$. $J=15.03 \mathrm{~Hz}), 7.33(5 \mathrm{H} . \mathrm{m}), 6.24$ (1H. d. $J=15.03 \mathrm{~Hz}), 3.97$ (2H. t. $J=7.29 \mathrm{~Hz}) .3 .14(2 \mathrm{H}$. t. $J=8.01 \mathrm{~Hz})$ and $2.12(2 \mathrm{H}$, m) ppm. ${ }^{13} \mathrm{C}$ NMR $\left(75 \mathrm{MHz}, \mathrm{CDCl}_{3}\right): \delta 200.76 .135 .40$, $128.79,127.61 .126 .32$. 126.18. 117.68, 52.32, 45.29 and $19.53 \mathrm{ppm}$. Infrared (neat): 3045.9 . 2985.6-2892.0. 1636.3. 1476.7. 1457.4, 1431.9. 1413.0, 134.1, 1287.8. 1258.7. 1136.6. $949.5,763.0$ and $699.4 \mathrm{~cm}^{-1}$. FAB MS, $\mathrm{m} / \mathrm{s} 203.1\left(\mathrm{M}^{-}\right)$.

Acknowledgements. This research was supported by the Kỵungsung University Research Grants in 2006.

\section{References}

1. (a) Barros, J. A. G; Fechine. G. J. M.: Alcantara, M. R.; Catalani. L. H. Polmmer 2006. $47(26)$. 8414-8419.

2. Movassaghi. M.: Huntt. D. K.: Tjandra. M. J. Ant Chent. Soe 2006. $128(25) .8126-8127$

3. Iddon1. B.: Tonder. T. E.: Hosseini. M.: Begtrup. M. Tetrohedon 2007. 63(1), 56-61

4. (a) Golier, F.: Boulhadjera. K.: Faye D.: Gaulon. C.: Maisonneuve. V.: Dujardin. G.: Dhal, R. Org. Lett. 2007. 9(2). 211-214. (b) Savitha. G.: Perumal. P. T. Tetrahedron Lett. 2006. $+7(21) .3589$ 3593. (c) Han. B.: Jia. X.: Jit1. X.: Zhou. Y.: Yang. Li.: Liu. Z.: Yu. W. Tetrohedhon Lett. 2006. $+7(21) .3545-3547$.

5. Hansen. A. L.: Skrydstrup, T. Org. Lett. 2005. 7(25). $5585-$ 5587 .

6. Movassaghi, M.: Hill, M. D. J. Am. Chem. Soc. 2006, 128(44). 14254-14255.

7. Shimasaki. Y.: Yano. H.: Ariyoshi. K: Kambe. H. J. Mol Cat .A: Chent 2005. $239(1-2) .125-129$.

8. Gohier. F; Boulhadjera, K; Faye, D.: Gaulon. C.: Maisonneuve. V.: Dujardin. G.: Dhal. R. Org. Lett. 2007, 9(2). 211-214.

9. Taillefer, M.: Ouali. A.; Renard, B.: Spindler, J. Chem. Ew: $d$. 2006. $12(20)$. $5301-5313$.

10. (a) Movassaghi. M.: Ondrus. A. E. J. Org. Chent 2005. $70(21)$. 8638-8641. (b) Lebedev. A. Y.: Izmer. V. V.: Kazyul'kin. D. N.: Beletskaya, I. P.: Voskoboynikov. A. Z. Org. Lett. 2002. f(4). 623626.

11. Sun. C.: Camp, J. E.; Weinreb, S. M. Org. Lett. 2006. $8(9), 1779$. 1781.

12. Flowers. R. G.: Miller. H. F.: Flowers. L. W. J. Ant. Chem. Soc. 1948. $70.3019-3021$

13. (a) Perreux. L.: Loupy. A. Tetrahedron 2001, 57, 9199-9223. (b) Kappe. C. O. Angew. Chem. Ih. Ed 2004, 43. 6250 and references therein. (c) Kuhnert, N. Angew: Chem. hit. Ed. 2002. H. 1863. (d) Strauss. C. R. Angew. Chen. Int. Ed 2002. H. 3589. (e) Choi. J. Y.: Kim. J.: .hung. S. H.: Kim. H.-K.: Chang. J.S.: Chae. H. K. Bull. Kot Chent. Soc. 2006. 27(10). 1523-1524.

14. (a) Kim. J. K.: Kwon. P. S.; Kwon. T. W: Chung, S. K : Lee J. W. Swn. Commun. 1996, 26, 535. (b) Kim. S. Y.; Kwon. P. S.: Kwon. T. W. Chung, S. K. Chang. Y. T. Syn. Commm. 1997, 27. 533 . (c) Kwon. P. S.: Kim. Y. S.: Kang. C. T.: Kwont. T. W.: Chung. S. K.: Chang. Y. T. Swn. Commun. 1997. 27. 4091. (d) Loupy. A.: Song. S. T.: Sohn1. S. M.: Lee. Y. M.: Kwonl. T. W. J. Chem. Sac. Perkin Trans. 12001.12201222.

15. Kim. K. W.: Alm. H. S.: Lee. H. J.; Song, S. J.: Kim, C. G.: Kwon. T. W. Bull. Ko: Chem. Soc. 2006, 27(2). 286-290. 
16. Kwon. P. S.: Kim. J. K.: Kwon. T. W.: Kim. Y. H.: Chung. S. K. Bull Kor. Chem. Soc. 1997. 18(10). $1118-1119$.

17. Song. S. J.: Cho. S. J.: Park. D. K.: Kwon. T. W.: Jenekhe. S. A. Tetrahedron Lett. 2003, $4+(2) .255-257$

18. (a) Kwon, T. W.: Kwon. P. S.: Song. S. I.: Park. M. S.: Choi J. W. Bull. Kor Chem. Soc. 2002, 23(6). 917. (b) Park, M. S.: Choi, I. W.: Lee. J. H.: Kwon. P. S.: Kwon. T. W. Bull. Kor Chem. Soc. 1998. $19(8) .883$

19. (a) Kwon. T. W.: Keusenkothen. P. F.: Smith. M. B. J. Org. Chem. 1992, 57(23). 6169-73. (b) Smith, M. B.: Wang, C. J.: Keusenkothen. P. F: Dembotsky. B. T.: Fay J. G.: Zezza. C. A.: Kwon, T. W.: Sheu, J: Son. Y. C.: Menezes, R. F. Chem. Lett.
1992. 2. $247-50$

20. (a) Arthur. S. H.: Ruth. B. K.: Toseph. P. M. Tetrahedron Lett. 1983. $2 f(8) .829-830$. (b) Albert. P.: Martin. N. T.: Andreas. S. $J$ Org. Chem 2004. 69, 33-45. (c) Dawei. M.: Haiying. S. Tetrahedron Lett. 2000. H. 1947-1950. (e) Vedejs, E.: Wilde. R. G. J. Org. Chem. 1986. $51(1), 117-119$.

21. (a) Hussaini. S. R.: Moloney. M. G. Ong \& Bionol. Chem. 2006. f(13). 2600-2615. (b) Yde. B.: Yousif. N. M: Pedersen. U.: Thomsen. I.: Lawesson1. S. O. Tetrohedon 1984. fo(11). 2047-52

22. Pinnick, H. W. Chang, Y.J. Org. Chem. 1978. $43(24)$. $4662-4663$.

23. Loupy, A. In Mficow ares in Organic Symthesis. $2^{\text {nd }}$ ed: WileyVCH: Weinheim (Germany). 2006. 